\title{
The Phonetic Anamorphoses-Oral Tori and Exostoses
}

\author{
Anubha Bajaj* \\ Deputy General Manager, Indiass
}

Received: May 17, 2018; Published: July 19, 2018

*Corresponding author: Anubha Bajaj, Deputy General Manager, LT Foods Ltd, LT Group Company (Daawat Basmati Rice), India, Email: anubha.bajaj@gmail.com

\section{Mini Review}

Tori and Exostoses are localized, peripheral, benign bone protuberances of obscure aetiology, arising from the cortical plate. The solitary lesions can be nodular, flat or pedunculated, detached bony outgrowths in the young, dentate subjects, localized on the jaw/alveolar bones [1], infrequently presenting in the person as multiple exostoses. The molar/ bicuspid zones are the affected sites of the jaw [2], though other sites are known. Anatomic conformations of the established oral exostoses, as per location, are alluded to in the maxilla as (Torus Palatines TP) a sessile nodular bony lump perceived on the midline of the hard palate, and in the mandible as (Torus Mandibular is TM) a bony protuberance on the lingual aspect of canine and premolar mandible [3].

The lesion usually elucidates as a smooth, bulging bone surface continuous with the adjacent area or as discreet, multilocular spherical projections with a broad based nodular cluster $[4,5]$. Multivariate ethnic groups elucidate a prevalence rate of $0-66 \%$ for TP and 0-85.7\% for TM. Buccal Bone Exostoses (BBE) is a benign, broad based external mass positioned on the maxilla or sporadically on the mandible, in the ratio of 5.1:1 correspondingly. The self-limiting, painless lumps commence in early adulthood and enlarge gradually. Buccal exostoses implant only on the alveolar bone usually in the maxillary alveolus [6]. Buccal exostoses may handicap the prosthodontic dental insertion. Buccal exostoses can be traumatized and interfere with oral hygiene and though infrequent, may impinge upon the jaws. The mucosal projection is intact and unremarkable; the nodules are dense and bony on palpation. Mucosal ulceration that is visualized is due to the traumatic laceration.

Torus mandibular is perceived as a solitary, mandibular premolar bony ridge or as clustered bony lumps on the lingual, alveolar border. The torus can be bosselated or multilobulated, but exostoses typically is a single, broad based smooth mass with a central pointed, tender bony projection. Exostoses originate during the teen years and subsequently amplify. Innocuous and self-restricting bony lumps portray buccal exostoses. Periodontal disease of the contiguous teeth is attributed to the ingested food and enlarged exostoses. Coexistent periodontal conditions, pain, distress, lump above the lip obligate treatment. Exostosis is a condition of obscure aetiology where the formation of TM, TP and exostoses is contributed by identical, formative aspects. However, exostoses are hypothesized to be of a multifactorial aetiology including functional prominence (masticatory hyper functioning) and persistent evolution of the jaw bones. Racial, genetic with autosomal dominant penetration, dental attrition and nutritional factors are implicated in the aetiology. Concurrent multiple exostoses and tori usually symbolize a multiple exostoses syndrome. Bony exostoses or tori elucidate therapy in cases of dentine installation, hampered periodontal hygiene and with reoccurring traumatic ulcerations. Exostoses can accomplish a buttressing purpose to counter the disproportionate force of dental occlusion. Edentulous patients with a diminished muscular power may remodel and eradicate the exostoses [7] (Figure 1).

\section{Tori and Exostoses}

- Tori and exostoses are protuberances of dense cortical bone that most commonly seen in adults.

- characterized by slow growth that usually arrests spontaneously

- usually manifest with no symptoms, except in the case of trauma, and they may complicate denture fitting.

- buccal exostosis- arises from the buccal cortex of the maxilla

- torus mandibularis- arises above the mylohyoid line, along the lingual surface of the mandible

- torus palatinus- arises from the midline hard palate; and - torus maxillarus-which arises from the lingual surface of the posterior maxilla

Figure 1: Courtesy: Slide share.

\section{Clinical peculiarities}

The maxilla at the premolar/molar zone is implicated in the bilateral lumps which lack specific history/symptoms. Discomfiture, alcohol/drug/tobacco consumption, co morbid 
conditions, family history or allergies are non-existent. Genetically predisposed population with operational irregularities may augment the evolution of exostoses as doe's prominent dental attrition in the elderly [8]. Maxillary molars at the buccal zone demonstrate the enlarged, bilateral masses. The mucosa superimposed on the palpably dense bony lumps is thin. Exostoses which are oblong and sharp-edged do not hinder the speech, mastication and added oral objectives.

\section{Radiology}

Premolars and molars may explicate a well demarcated radio opaque area with or without residual rests. The delineation of bilateral buccal exostoses (BBE). is aided by augmented, condensed cortical plates on the maxillary buccal exterior and do not, necessitate therapy. Surgically, bilateral (horizontal/ vertical) micro periosteal flaps are reversed under local anaesthesia to incise the exostoses with a continually irrigated, bone cutting carbide bur. A bone file is utilized for developing a frictionless exterior. Granulation tissue is curetted. Chiselled, marginal bone is analysed.

\section{Histopathology}

As the nomenclature indicates the extricated marginal bone chips displays native bone (Figure 2).

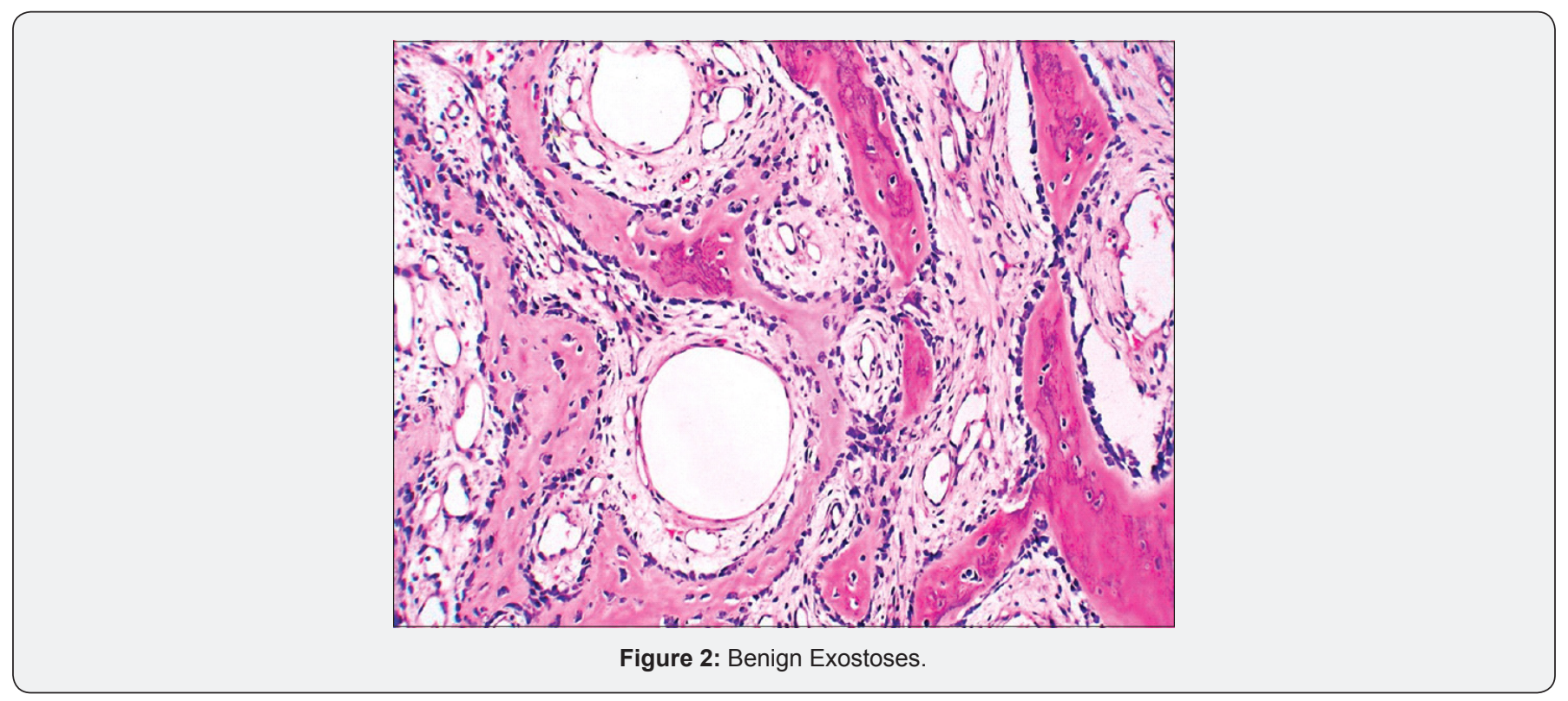

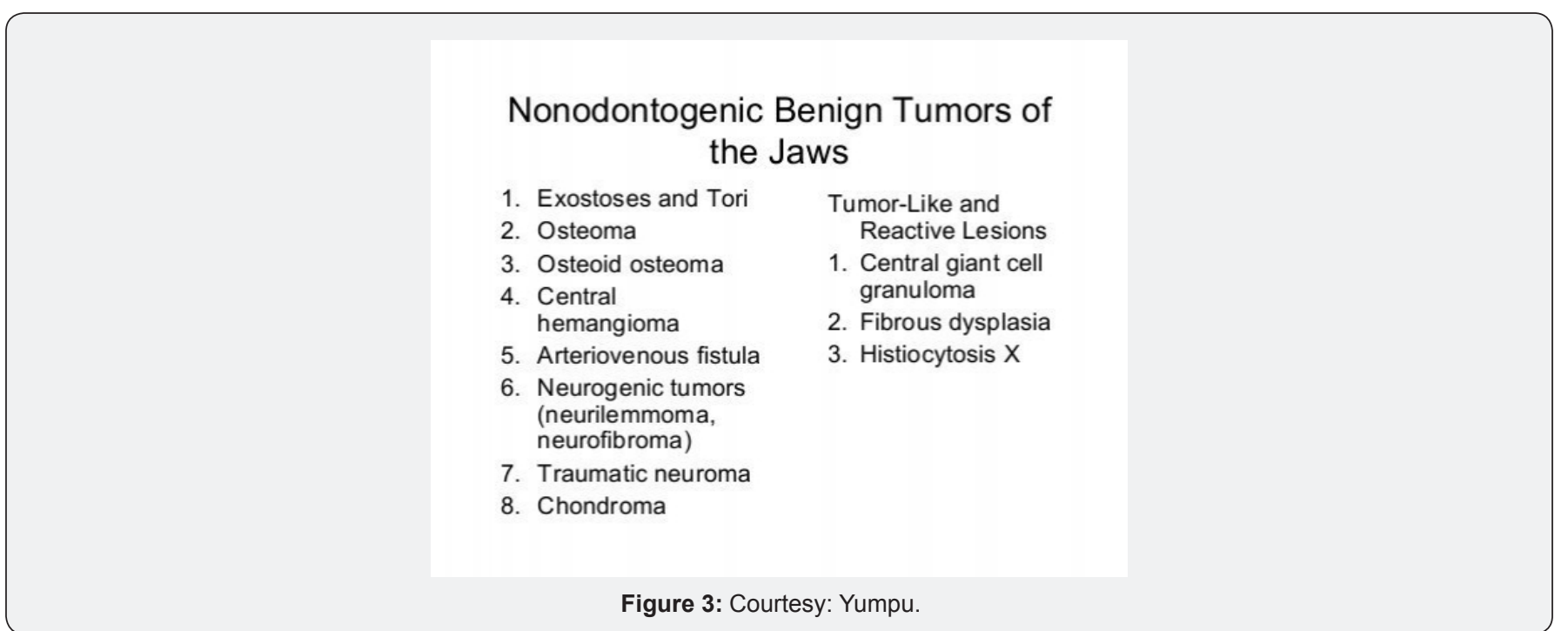

\section{Colloquy}

A benign, peripheral, confined bony outgrowth of obscure aetiology impinging upon $3 \%$ of the preponderantly male adult population. Males are frequently afflicted in contrast to the females proportioning at 1.66: 1 at all intraoral sites.
Exostoses are a nodular, flat or pedunculated excrescence localized on the alveolar covering of the contiguous jaw bones [9]. Oral functional adaptation induces the exostoses in a genetically inclined population [10,11]. It is challenging to detect the true origin of the exostoses as perpetual progress may be visualized. Buccal exostosis is distinguished by the 
clinical essay and the radiography. Clinically, the tori arise as multiple, rounded excrescences or lobular calcifications, while the exostosis is a solitary, broad based, mucosa cloak a pointed bony extension. Exostoses demands distinction from compact osteoma, cancellous osteomas, osteochondromas, organized subperiosteal haematoma, mature ossifying fibroma (expanding cortical lamina), periosteal osteosarcoma or chondrosarcoma. Osteomas are benign neoplasms which activate proliferation of dense, compact or coarse cancellous bone generally in an endosteal or periosteal location $[12,13]$. The developmentally aberrant neoplasm has analogous clinical, radiographic and histological characteristics. Dense compact or cancellous bone propagates in the benign tumour. Numerous bony outgrowths or lumps in atypical locations should arouse the suspicion of Gardner's Syndrome which comprises of intestinal polyps, cutaneous cysts or fibroids as components of the autosomal dominant syndrome. Diagnostic clarification should be sought with histopathology neither the torus nor the bony exostoses required treatment unless it became large enough to interfere with the normal function. Repeated traumatic external ulceration or enlarged tori/ exostoses entail treatment. The native bone of tori/exostoses can be employed as an effective donor site for an auto graft. Enormous maxillary exostoses and protrusions are appropriate substitutes for a cortical/cancellous autologous graft. Intra-operative challenges and post-operative complications are diminished. Centralized alveolar ridge defects can be corrected. Specific autogenous and block bone can be obtained from the lesion. Elective therapy employs a bone cutting bur to annihilate the bony mass or chisel the base of the lesion, if surgical treatment is not an option, as in edentulous patients. Alternatively, denture can be alleviated to minimize mucosal laceration [14].

\section{Corollaries}

Buccal bone exostoses (BBE) are a superior donor substitutes for a bone locus for autologous graft in addition to the symphysis and mandibular ramus. Localized bony irritation underneath the free gingival and skin grafts can abet and energize the composition of solitary exostoses with new bone synthesis (Figure 3).
Your next submission with Juniper Publishers will reach you the below assets

- Quality Editorial service

- Swift Peer Review

- Reprints availability

- E-prints Service

- Manuscript Podcast for convenient understanding

- Global attainment for your research

- Manuscript accessibility in different formats ( Pdf, E-pub, Full Text, Audio)

- Unceasing customer service

Track the below URL for one-step submission https://juniperpublishers.com/online-submission.php 